\title{
MENINGOCOSIS DEL APARATO LOCOMOTOR
}

\author{
Por el Dr. HELMUT V'. JAEGER \\ Sexción Cirugía, Ortopedia y Tramatologia alel Hospital de Niños "Manuel \\ Arríará" (Jefe: Dr. A. Inostrosa) \& Instituto Traumatológico \\ (Jefe: Prof. T. Gebauer)
}

E1 presente trabajo tiene por objeto relatar nuestra experiencia sobre las localizaciones de la infección meningocócica en el aparato locomotor que se han presentado en los niños hospitalizados en el Servicio de Infecciosos (Dr. A. Steeger) de la Sección Medicina (Prof. E. Cienfuegos) del Hospital "Manuel Arriarán" desde el $1 .^{\circ}$ de enero hasta el 31 de octubre de 1942. En el transcurso de esos 10 meses han pasado por aquel Servicio 1.000 casos de infección meningocócica. En 37 de estos niños se hizo el diagnóstico clínico de artritis meningocócica. ninguno de ellos falleció (Cuadro 1). Pero en 5 de 30 casos que llegaron al Servicio de Anatomía Patológica del Hospital (Dra. E. Peña) y en que se abrieron sistemáticamente las articulaciones de la rodilla, se encontraron sinovitis supuradas francas, con dertame purulento a veces abundante, no diagnosticadas intra vitam. En 4 de estos 5 casos el examen bacteriológico reveló meningococos, si bien no le damos un valor absoluto a estos exámenes, por no haberse extraído t'l pus en condiciones estrictamente asépticas. Posteriormente, en 10 casos, abrimos personalmente la totalidad de las grandes articulaciones (escápulohumeral, codo, muñeca, coxofemoral, rodilla. tibiotarsiana) encontrando sinovitis purulentas múltiples en 4 casos y en 2 de ellos inflamaciones purulentas de las bolsas serosas y de las vainas serosas peritendinosas (Cua-

(*) Revirado y completado en junio de 1943. Originalmente publisado en Ios Arebioos de la Sociedad de Cirujanos de Horpital. 12.1305 .318 (งiciembre) 1942. 


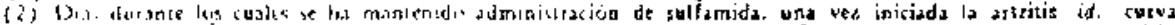

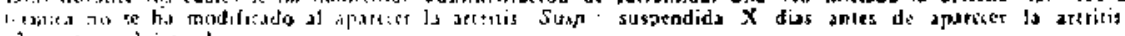

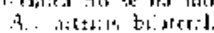


dro 2), lesiones de las cuales no hemos encontrado mención en la literatura revisada.

Ulteriormente. hasta fines de junio de 1943, hemos abierto la totalidad de las grandes articulaciones en 12 nuevos casos, encontrando sinovitis putulenta en 4 , lo que hace un total de 52 autopsias con 13 casos con hallazgos positivos.

En la mayoría de los grandes tratados tanto de medicina. cirugia u ortopedia, cuanto en las monografías sobre infección meningocócica, las localizaciones en el aparato locomotor sólo se mencionan de paso, para decir que se presentan con tal o cual frecuencia y que su pronóstico es relativamente benigno. La magnitud y la multiplicidad de estas lesiones merecen, sin embargo, un estudio más detenido.

Para tener una idea clara de la etiopatogenia de las meningocosis del aparato locomotor. es preciso decir dos palabras sobre él.

Concepto actual de infección meningocócica o meningocosis.-El concepto patogénico y anatomoclínico sobre la meningitis meningocócica o cerebroespinal epidémica o fiebre cerebroespinal. ha evolucionado en los últimos años. Hace 2.5 años aun se aceptaba que el meningococo llegaba a las meninges por las prolongaciones del nervio olfatorio o a través de los linfáticos del nasofarinx. Actualmente, existe acuerdo casi unánime en el sentido de que el meningococo, partiendo de la nasofaringe, invade el torrente citculatorio produciendo una septicemia meningocócica o meningococemia, fijándose secundariamente en diverscs órganos y tejidos y en forma preferente y casi obligada en lás meninges. Las lesiones sinoviales purulentas precoces que hemos encontrado, en la mesa de autopsías son la mejor demostración de esta septicemia inicial. E1 hallazgo de estas lesiones purulentas diseminadas precoces es de gran trascendencia patogénica, pues demuestra que estas "complicaciones" no aparecen "por lo general en la segunda semana de evolución" ni son "manifestaciones tardías de un daño provocado al iniciarse la enfermedad o el resultado de la acción tóxica o alérgica de la infección". como se ha venido repitiendo hasta hoy día (octubre 1942), sino que en muchos casos son localizaciones hematógenas precoces. simultáneas a la invasiôn meníngea.

Los síntomas que se instalan a consecuencia del compromico meníngeo dominan en tal forma el cuadro clínico, que fácilmente se olvida el carácter septicémico de la afección. Y sólo teniendo presente que la meningitis no es más que una 
CUADRO 2,-MENHGOCOSIS DEI, APARATO LOCOMOTOR DIAGNOSTICADAS POSTMORTEM. - CAUSA DE MUERTE: LEPTOMENINGITIS MENINGOCOCICA. CASUISTICA PERSONAL.

\begin{tabular}{|c|c|c|c|c|c|c|c|c|c|c|}
\hline & & & & 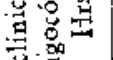 & 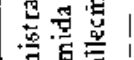 & & Sinovitis & purulentra & & \\
\hline$\dot{z}$ & & $\ldots$ & $\begin{array}{l}0 \\
\ddot{3} \\
心\end{array}$ & 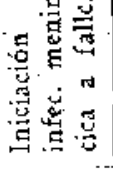 & 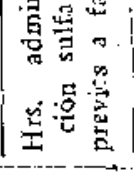 & 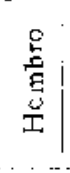 & 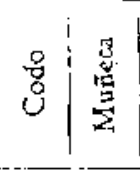 & 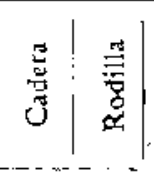 & $\stackrel{0}{\stackrel{0}{E}}$ & $\begin{array}{l}\text { Bursitis y } \\
\text { tendosinovitis }\end{array}$ \\
\hline 1 & 2 & a & $\mathrm{m}$ & 67 & 22 & & & I & & \\
\hline 2 & 8 & a & m & 56 & 18 & & & A & & \\
\hline 3 & 2 & 2 & $m$ & 54 & 32 & & & $\mathrm{D}$ & & \\
\hline 4 & 6 & a & $\mathrm{m}$ & 35 & 6 & & & $D$ & & \\
\hline 5 & 2 & a & $f$ & 94 & 64 & & & 1 & & \\
\hline 6 & $1 \%$ & $\mathrm{~m}$ & $-m$ & 40 & 4 & - & $-A$ & I & $\mathrm{D}$ & \\
\hline 7 & 1 & $\mathbf{a}$ & $\mathbf{m}$ & 97 & 25 & $A$ & A $A$ & - $A$ & I & $\begin{array}{l}\text { B. sabdeltoidea I. T. } \\
\text { extensores dedos D. } \\
\text { y tibial posterior D. }\end{array}$ \\
\hline 8 & 7 & a & $\mathrm{t}$ & 72 & 48 & - & $-\cdots$ & $\div-$ & $\mathrm{D}$ & \\
\hline 9 & 5 & $a$ & $\mathbf{m}$ & 40 & $1 \mathrm{I}$ & - & A - & $-A$ & $\mathrm{D}$ & $\begin{array}{l}\text { T. peroneos latera. } \\
\text { les bilateral. }\end{array}$ \\
\hline 10 & 5 & $\mathbf{m}$ & $\mathrm{m}$ & 48 & 19 & - & $-\cdots$ & - & $\mathrm{D}$ & \\
\hline 11 & 6 & a & $\mathrm{m}$ & $3 \dot{3}$ & 3 & 1 & -- & $A$ & - & \\
\hline 12 & 1 & $a$ & $\mathrm{~m}$ & $?$ & 54 & D & A 1 & $-\quad-$ & - & \\
\hline 13 & 3 & $\mathbf{a}$ & $f$ & 39 & 9 & - & $-A$ & -- & I & $\begin{array}{l}\text { B. subescapular D. } \\
\text { comuaicada coat es- } \\
\text { cápulo-humeral a } \\
\text { través del fotamen } \\
\text { oval de Weitbrecht. }\end{array}$ \\
\hline
\end{tabular}

localización de una infección mucho más generalizada, se podrá comprender la patogenia y la clínica de la enfermedad. Es así como hoy dia se habla de infección o enfermedad meningocócica y no de meningitis meningocócica, para designar la enfermedad en su totalidad. Como la denominación infección. meningocócica es larga y engorrosa, crémos que es más cómo- 
do, sin dejar de ser correcto, reemplazar este término por el de meningocosis, equiparándolo a los de tuberculosis, equinococosis, neumoconiosis, etc., y englobando bajo esta denominación todas las localizaciones ya meníngeas ya extrameníngeas de la infección meningocócica. Si preferimos este término al ya existente de meningacoccia, es porque creemos que la desifnencia osis acentúa más y graba mejor la idea de enfermedad con localizaciones múltiples. Para las localizaciones meníngeas se reservaria la denominación "meningitis meningocócica" o meningocosis meníngea y las localizaciones extrameníngeas se designarían según el órgano comprometido, ar.tritis, endocarditis, bursitis meningocócica, etc., o meningocosis articular, endocárdica, de las bolsas serosas, etc. Decimos "localizaciones" y no "complicaciones extrameningeas" para recalcar que son procesos paralelos y no secundarios a la localización meningea.

La gravedad que la localización meníngea imprime al cuadro clínico hace que la infección meningocócica se clasifique según la evolución de aquélla, distinguiéndose así distintas formas clínicas que pueden resumirse en tres:

1. ${ }^{\circ}$ Forma fulminante o maligna;

2.: Forma aguda común, y

3.9 Forma septicémica crónica.

1. ${ }^{\circ}$ La forma fulminante o maligna termina generalmente en horas con el enfermo, 8 a 60 , y la marcha paede ser tan rápida que no alcancen a comprometerse las meninges. En reIación con el compromiso o la indemnidad de las meninges, se decriben dos tipos, tipa septicénico, sin compromiso meníngeo, con síndrome hemorrágico intenso de piel y mucosas, que al comprometer las supratrenales constituye el síndrome de Waterhouse-Fridrichsen, y tipo nervioso, en que la violencia de la infección cae sobre el encéfalo.

2.\% La forma aguda común abarca cerca del $90 \%$ de los casos de infección meningocócica y constituye la meningitis meningocócica propiamente dicha, de todos conocida.

3." La forma septicémica crónica es una septicemia leve. con pcca tendencia al compromiso meníngeo, que evoluciona durante semanas o meses, con brotes febriles y localizaciones diversas y cuyo diagnóstico muchas veces ni aun con el hemocultivo logra precisarse.

Con el uso de las sulfamidas se ha observado una modi. ficación evidente del cuadro clínico y de la evolución de la infección meningocócica, que sólo queremos enunciar. 


\section{Meningocosis del aparate locomotor}

Hemos elegido este título para englobar bajo un común denominador las localizaciones de interés quirúrgico-ortopédico de la infección meningocócica, es decir, las artritis, desde tiempo conocidas, las bursitis y las tendosinovitis purulentas, hasta ahora aparentemente ignoradas. A estas tres focalizaciones habria que agregar aún las osteomielitis meningocóricas, de las cuales sólo hemos encontrado referencias en la literatura y los nódulos aponeuróticos dolorosos, que según Dénis Brinton, tendrian generalmente el volumen de una atveja, aparecerían del $4 .^{\circ}$ al $6 .^{\circ}$ día de enfermedad y sólo perdurarian algunos días. Cuando su localización es subcutánea, trasluciría a veces el color. rojizo oscuro hemorrágico simulando el eritema nudoso.

Decimos "meningocosis del aparato locomotor" y no "meningocosis quirúrgicas", porque esta última denominación frejuzgaria en lo que a terapéutica se refiere, si bien ella podría abarcar los pocos casos de peritonitis y orquiepididimitis meningocócica que se registran en la literatura y que con la medicación sulfamidada parecen ser cada vez más rarcs.

La frecuencia con que se han observado clinicamente las diversas localizaciones extrameningeas en los 1.000 casos de infección meningocócica que han pasado por el Hospital Arriarán en los 10 primeros meses de 1942 fué la siguiente:

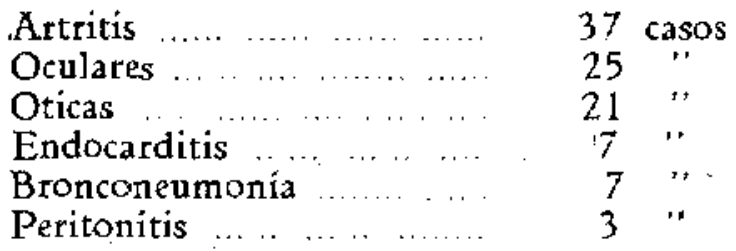

De este cuadro se desprende que la localización articular ha sido la más común.

I. Bursitis y tendosinovitis supuradas.

Estas localizaciones, de las cuales no hemos encontrado mención en la literatura, han sido un hallągo de autopsia. Encontramos casualmente en un niño de 1 año 5 meses. fallecido 97 horas después de iniciado el cuadro clínico, además de una leptomeningitis menincogócica y de múltiples artritis 
supuradas, una colección purulenta. pus amarillento verdoso espeso, en la bolsa serosa subdeltoidea izquierda, sin comunicación con la cavidad sinovial escápulo-humeral y existencia de pus de igual aspecto en las vainas tendinosas carpianas de los extensores de los dedos y del tibial posterior derecho. En a cegundo caso se trataba de un niño de 5 años, que falleció 40 hcras, después de iniciado el cuadro clínico de infoción meningocócica. En la autopsia encontramos junto a artritis purulentas múltiples tendosinovitis purulenta bilateral de los peroneos laterales. La capa interna de las bolsas serosas y de las vainas tendinosas mostraba alteraciones macroscópicas muy discretas: sólo escasa congestión vasiular y ligero deslustramiento.

Podría argüirse que el proceso podría haberse propagado. a las bolsas: serosas y vainas tendinosas desde las articulaciones adyacentes. Pero en el primer caso existía una tendosinovitis tibial posterior derecha y en el segundo de los peroneos laterales izquierdos.y en ambos casos tanto las articulaciones tibio-tarsianas cuanto las subastragalinas homolaterales estaban indemnes (*).

Estas lesiones pueden explicar numerosos casos que antes interpretábamos como artritis, en los cuales encontrábamos aumento de volumen doloroso que no correspondía exactamente a las interlíneas articulares. como, por ejemplo, en el dotsc de la mano o en sólo una de las regiones maleolares, externa o interna y en los cuales la limitación de la movilidad áricular era discreta o nula.

La evolución aparentemente es siempre benigna, ya que rasta abora no hemos observado secuelas que puedan atribuírceles y el tratamiento no será nunca quirúrgico.

\section{Artritis meningocócicas.}

Englobamos bajo este titulo todas las manifestaciones articulares de la infección meningocócica.

Las artritis meningocócicas ya fueron descritas por $T$. Welch en $1810 \mathrm{y}$ por $\mathrm{E}$. North en 1811 , mucho antes que Weichselbaum descubriera el meningococo (1887). G. $F$. Still (1898) y N. B. Gwyn (1899) ratificaron la naturaleza meningocócica, aistando el germen del líquido atticular extrái-

(*) En febrero de 1943 encontramos en un caso una gran colección purvlenta de la bolsa serosa subescapular, en comunicación con una sinovitis purulenta escápulo-humeral, a trapús del foramen oval de Weitbrecht. 
CLADRO 3.-FRECUENCIA DE LAS ARTRITIS .MENINGOCOCICAS

$$
\text { Autor }
$$

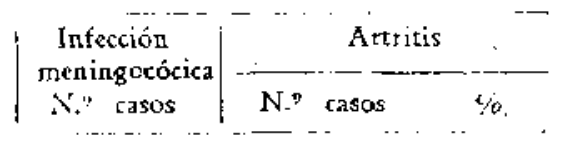

Councitman, Majlory y Wright 1898

5,4

Sophian 1913

10 a 115

Netter y Durand 1915

Fairley Y Steward 1916

Roger 1918

Rolleston 1919

Sainton 1919

\begin{tabular}{|c|c|c|}
\hline \multirow[t]{2}{*}{323} & 23 & 7 \\
\hline & & $5 \backsim 20$ \\
\hline 502 & 24 & 4,8 \\
\hline & 17. & 20 \\
\hline 321 & & 6.5 \\
\hline 144 & 11 & 7,6 \\
\hline $\begin{array}{r}368 \\
\text { sucto }\end{array}$ & 10 & 2.7 \\
\hline $\begin{array}{c}106 \\
\text { ulfamio }\end{array}$ & 5 & 4.6 \\
\hline
\end{tabular}

Herrick y Parkhurst 1919

Smitbburn et allii 1930

2:3

Schein $19 \geq 8$

1938

$$
\text { sulfamida }
$$

Bolda:an 1938

169

7.7

Brinton 1940

Hodes y Streng $19+2$

$110^{\cdots---5}$

Horwitz 1942

471

20

4.

Jaegzr 1942

1,000

4,2

Alessandri 1942

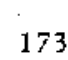

Horwitz 1942

1,500

37

+ de 3.7

8

9.5

do por punción y W. Osler en 1899 le dedicó un articulo en el British Medical Journal ( ${ }^{+}$Arthritis of Cerebrospinal Fever"). Posteriormente se publicaron numerosos casos clinicos aislados y sólo la primera guerra mundial dió origen, con sus grandes epidemias militates, a nuevos trabajos de conjunto: 
de Sir Humpbry Rolleston, en Inglaterra; de Herrick y Parkhurst, en EE. UU., y de Paul Sainton, en Francia. De los trabajos posteriores cabe destacar el publicado por Albert Schein, ortopedista de Nueva York, en 1938, en los "Archives of Internal Medicine".

Frecuencia.-Schein, revisando las observaciones de 10 años de 2 hospitales de Nueva York (Bellevue y Mont Sinai) sólo logró reunir 23 casos, 2 de los cuales eran comunicacicnes personales. Estas cifras hacen resaltar la magnitud de nuestra casuística, que alcanza a 37 casos en 10 meses.

La frecuencia que se le asigna a las artritis en la infección meningocócica, varía en la literatura, a través de varias epidemias de distintas épocas entre el 2.7 y el $20 \%$ (Cuadro 3 ). Sainton, que da el porcentaje más alto, cree que algunas artritis puedan pasar inadvertidas en casos de grave compromiso del estado general; por consiguiente, la frecuencia sería aún mayor. Esta suposición de Sainton tha sido confirmada por ncsctros. En 52 formas fulminantes que llegaron a la mesä de autopsia y en las cuales no se había hecho el diagnóstico de artritis, la inspección sistemática de las articulaciones reveló artritis purulentas en 13 casos. Por consiguiente, la frecuencia real es muy superior a la frecuencia clínica de $3.7 \%$ que arroja nuestra estadística.

Anatomía patológica.--En las artritis meningocócicas las alteraciones morfológicas son semejantes a las que se encuentran en las restantes artritis agudas infecciosas específicas hematógenas.

Herrick y Parkhurst dividieron en 1919 , las artritis meningocócicas en tres grandes grupos, clasificación que aun as: aceptada por los autores americanos (Schein, 1938: Comrce, 1941) (Cuadro 4):

Tipo A: Forma hemorrágica poliartrítica precoz, premeningítica (con mayor frecuencia), artrálgica o "reumática".

Tipo B: Forma purulenta ordinaria: monoartrítica, postmeningitica; $y$

Tipo C: Forma serosa tardia.

Nosctros no aceptamos esta subdivisión en formas anátomoclinicas diversas, en tipo hemorrágico, purulento y seroso. Afirmamos que la artritis meningocócica. tal como otras artritis agudas infecciosas, es una artritis purulenta, en la cual las lesiones articulares y el cuadro clínico adoptan alguna de las formas evolutivas de ésta y ponemos en duda la existencia de 


\section{CUADRO 4. - CLASIFICACION DE IAS ARTRITIS MENIYGOCOCICAS} DE HERRICK Y PARK-HURST (TOMADO DE SCHEIN)

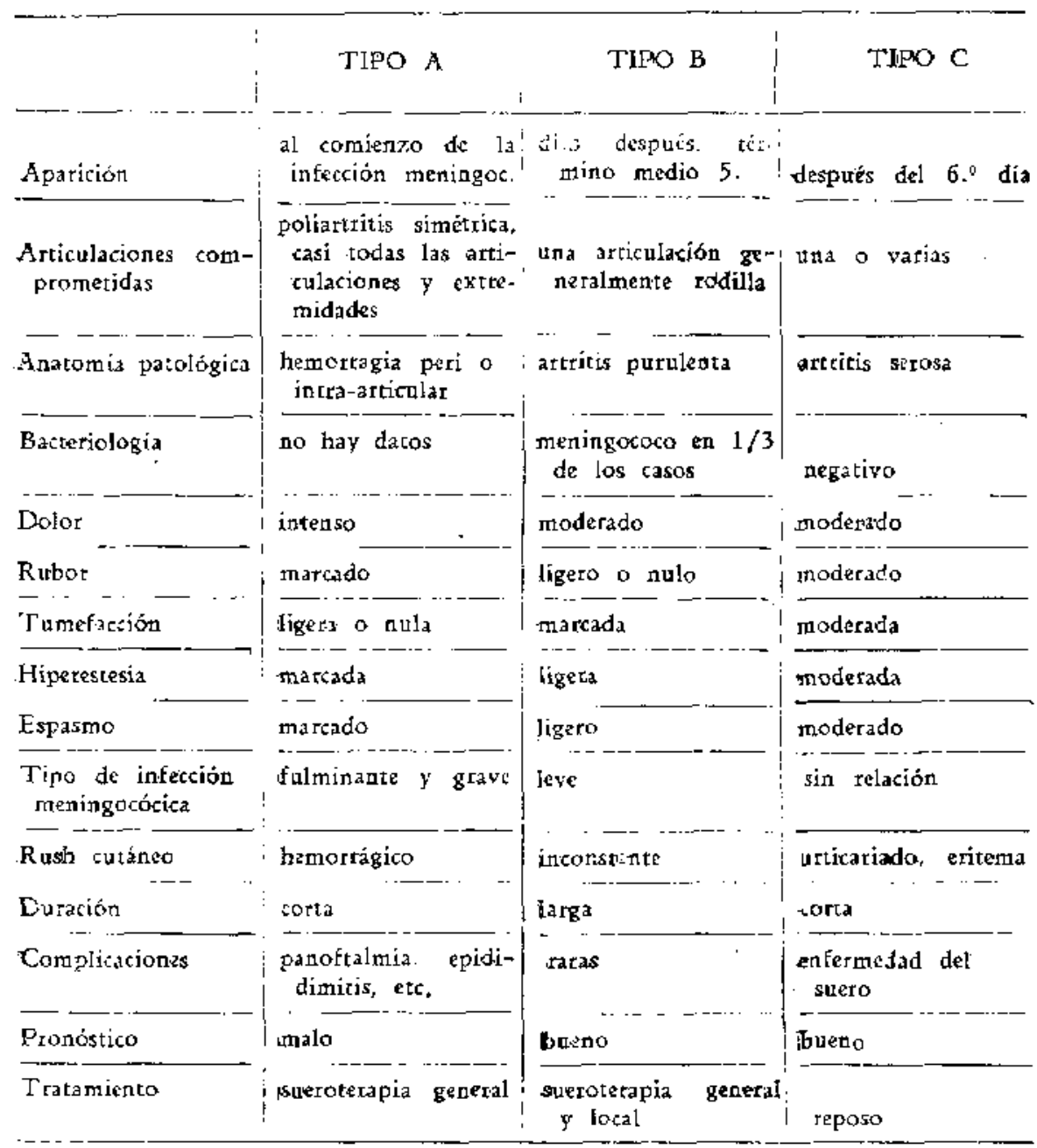

la forma hemorrágica precoz (tipo $A$ ) y de la forma serosa tardia (tipo C).

La clasificación de Sainton en tipos clínicos:

1." Tipo artrálgico;

2. ${ }^{9}$ Tipo supurado agudo o piartrosis meningocócica aguda; 
3.9 Tipo supurado subagudo o piartrosis meningocócica subaguda;

4." Hemartrosis; y

5.: Tipo plástico anquilosante,

No ha encontrado la aceptación de la de Herrick y Parkhurst y hoy día ya no se recuerda. Sainton consideraba el tipo supurado agudo como el más frecuente, del supurado subagudo había visto un solo caso, en el cual la evolución arrastrada hizo pensar en una tuberculosis de la muñeca, hasta que aparecieron signos meníngeos; el niño falleció y el examen microscópico del pus articular reveló meningococos.

Según Herrick y Parkhurst, en las artritis meningocócicas preccces (tipo A) existirían en los tejidos articulares focos hemorrágicos que irritarian la sinovial y qua podrían producir hemartrosis. Serían elementos similares a las erupciones purpúricas cutáneas. Virchow publicó el único caso de hemartrosis asociado a una meningitis, pero sin comprobación bacteriológica. En 52 casos fulminantes que llegaron a la mesa de autopsia no hemos encontrado jamás estas lesiones. pero sí artritis purulentas típicas. Ya Netter y Blanchier habian comprobado el mismo hecho pero evidentemente habia caído en el olvido. Estas artritis setían de corta duración y desaparecerian en general sin dejar vestigios. Sólo rara vez persistirían, adquiriendo entonces las características de la forma ordinaria (tipo B).

Consideramos que la forma serosa tardía (tipo C), que se describe como de carácter leve, evolución corta y buen pronóstico y que aparecería en la segunda semana de enfermedad. no es más que una vulgar artritis sérica, inherente al auge que en años anteriores tuvo el tratamiento con suero antimeningocócico. La artritis sérica, que aparece aproximadamente en el $14 \%$ de los casos de enfermedad del suero, 5 a 12 días después de colocado éste (desde minutos hasta 3 semanas o más en casos extremos), evoluciona generalmente con fiebre $y$ erupciones cutáneas. discreto dolor y a veces tumefacción y calor articular, desapareciendo espontáneamente en la mayoría de los casos.

Desde Still y Osler también se ha venido repitiendo que la inflamación sería casi siempre periarticular, es decir, que en la mayoría de los casos se trataría de periartritis, que las sinovitis supuradas serían excepcionales. Ya veremos que esto tampoco corresponde a la realidad. 
A las tres formas de Herrick y Parkhurst se agregaba aún una cuarta, la llamada espondilitis postmeningitica, aislada por S. Epstein (192). Epstein describió dos casos en los cuales existía una espondilitis productiva entre la tercera y cuarta vértebras lumbares. con adelgazamiento del disco intervertzbral. Creia que se trataba de una osteitis meningocócica hematógena metastásica. R. W. Billington, Profesor de Ortopedia en la Vanderbilt University, publicó en 1924 otros 35 cascs, en los cuales la sintomatología espondilitica apareció semanas a meses después de apagado el cuadro meríngeo. subdividiéndolos en tres grupos: $1 .^{\circ}$ Osteoartritis vertebrales con radicgrafías positivas: adelgazamiento del disco y exostosis marginales; 2. Casos con signos físicos de espondilitis, pero con radiografías negativas: y $3 .^{\circ}$ Casos con sintomatología lumbar, pero sin signos definidos de espondilitis. En sólo uno de sus casos se había presentado una artritis (muñeca) durante la evolución de la meningitis. Billington atribuyó estos cua. dros a la inoculación traumática directa del meningococo en los cuerpos o discos intervertebrales por el trócar, al hacer una raquicentesis intempestiva. Hoy dia es más lógico pensar en una hernia del núcleo pulposo, consecutiva a la lesión del disco intervertebral por el trócar, como ha sido observado por Levinson, 1919; Pease, 1935; Barr, 1937: Gellman, 1940; Munro y Harding. 1942, y otros.

Nosotros afirmamos que existe un solo tipo anátomoclínico de artritis meningocócica, en el cual el derrame y el compromiso tisular puede variar, adoptando alguna de las formas evolutivas de las artritis supuradas, purulentas o piógenzs. Sabemos que con Payr se distinguen dos formas: $\mathrm{em}$ piema articular y flegmón capsular. a los cuales se han agr?gado otras dos: osteoartritis supurada y flegmón paraarticular.

Si bien esta división es esquemática. ya que clínica y morfológicamente la transición muchas veces es gradual, es útil y didáctica para establecer las indicaciones terapéuticas.

El empiema articular o sinovitis puralesta de Volkmann es una inflamación exclusivamente sinovial, con lesiones discretas y superficiales de la capa interna o fibrocondroide de la sinovial, derrame generalmente abundante, temperatura moderada y buen pronóstico funcional. El flegmón capsular de Fayr o artritis supurada es más grave, la infección no sé limita a la cavidad articular, sino que invade la capa externa o celu. losa de la sinovial y la cápsula articular, en la cul se aprecia edema, trómbosis vascular e infiltrado inflamatorio, entendiéndose por cápsula con Payr todo el aparato cápsuló-liga- 
mentoso. El compromiso del estado general es intenso, hay edema e infiltración inflamatoria periarticular muy dolorosa $y$ cura con esclerosis y retracción tisular. es decir, con rigidez. $y$ aun anquilosis. En la ostecartritis supurada existe erosión y ulceración del cartílago articular, generalmente con compromiso del hueco subyacente. La radiografía revela pinzamiento articular, por destrucción condral y ósea. La anquilosis es la regla. En el flegmón paraarticular o fusión supurada, el tipo más grave, existe invasión masiva de las partes blandas adyacentes.

Las artritis meningocócicas son artritis purulentas. Los casos en los cuales hemos podido conocer la naturalezt del detrame por la punción articular $(5$ casos) revelaron siempre liquido purulento y tanto la sintomatología y evolución de estes casos cuanto de los restantes, encuadra dentro de las características de la sinovitis purulenta. En los casos en los cuales se evidenció el carácter purulento del derrame por punción articular, la artritis comenzó después del $60^{\circ}$ dia $170^{\circ}, 120^{\circ} \mathrm{y}$ 13. días), época en que según la clasificación de Henrick y Patkburst debería corresponder al tipo C, con líquido serosc. También en las 13 artritis que sólo constituyeron un hallazgo anátomopatológico, y que fallecieron 33 horas a 4 días después de iniciado el cuadro clínico de infección meningocócica, el derrame fué purulento y las lesiones sinoviales muy discretas: congestión vascular, pero no alteración evidente de la sinovial. En 2 casos en los cuales se realizaron cortes histopatológicos (Dra. E. Peña) el microscopio reveló sólo lesiones superficiales de la sinovial y discreta infiltración celular. Es decir, alteraciones del tipo del empiema articular. En ningún caso existían vestigios de hemorragia, en el sentido de Herrick y. Parkhurst (tipo A). En un caso de sindrome de Waterhouse-Fridrichsen, que llegó a la mesa de autopsia 14 heras de iniciado el cuadro clínico. se encontró en las partes blandas de la rodifla, tal como en los demás tejidos, una congestión vascular intensa, pero no signos de hemorragia.

En la literatura se encuentran algunos casos con lesiones articulares destructivas radiológicas, es decir. osteoartritis meningocócicas, que corresponden al tipo plástico anquilosante de Sainten y que en su mayoría terminaron con anquilosis (Cuadro 5), todas en adultos.

Cuadro clínico.--Jas attritis meningocócicas aparecen con más frecuencia entre el $4 .^{\circ}$ y $10 .^{\circ}$ dias. pero pueden aparecer ya el $2{ }^{\circ}$ día de iniciado el cuadro clínico de infectión meningocócica, ó 30 ó más días después. Seguramente la iṇi- 
ciación real, anátomopatológica. es mucho más precoz, al menos así lo demuestran los casos no diagnosticados clínicamenté, que constituyeron hallazgos de autopsia y en algunos de los cuales existía, sin embargo; un empiema articular apieciable. Si clínicamente no se hace un diagnóstico más precoz es porque el grave compromiso del estado general no permite al enfermo guiar por sus síntomas subjetivos la exploración clinica, por lo demás difícil en las lesiones atticulares incipientes.

Algunos han dividido las artritis meningocócicas, basados en su asociación $y$ relación cronológica con la meningitis $\mathrm{c}$ con otras localizaciones meningocócicas extrameníngeas. Así, por ejemplo. Sainton las clasificaba en:

1. Artritis meningocócicas aściadas a la meningitis meningccócica.-Pueden, a su vez, ser, pre, yuxta $\odot$ postmeningíticas. Las artritis premeningíticas (tipo $A$ de Herrick y Parkhurst) frecuentemente tomarian varias articulaciones, ptestándose así a confusión con el reumatismo poliarticular agudo. Sainton afirma haber observado un caso en que la meningitis sólo se manifestó a los 72 días de evolución de la artritis. Las artritis postmeningiticas pueden ser precoces $\left(4 .^{\circ}\right.$ a $7 .^{\circ}$ dias) o tardías $\left(13 .^{\circ}\right.$ a $35^{\circ}$ dias). Nuestros casos ban sido todos del tipo yuxta y postmeningítico.

2. Artritis meningacócicas asociactas a infectión meningcócica extrameníngea.-.No es más que una septicemia meningecócica crónica con localización preferente articular. una meningococemia sin meningitis, que da lugar a un "reamatismc." (Sainton) meningocócico. generalmente poliatticular poco intenso y pasajero. Estas formas evolucionan en general con curva febril y acompañados o no de otras localizaciones meningocócicas, tales como eritemas, herpes, bronceneumonías o iridociclitis. El diagnóstico etiológico, muchas veces sólo lo ha dado el hemocultivo o el desarrollo pesterior de una meningitis típica. En la literatura se encuentran múltiples casos aislados de este típo.

3. Artritis primaria o criptogenética.-La artritis constituye la única manifestación de infección meningocócica, ni antes ni después de la artritis aparecen otras localizaciones meningocócicas y el diagnóstico etiológico sólo lo podrá dar el laboratorio, demostrando la presencia del meningococo. De este tipo se han descrito sólo unos pocos casos (Cuadro 6). Naturalmente que en estos casos un cuadro meníngeo abortivo o una septicemia meningocócica leve puede fácilmente haber pasado inadvertida. 
CUADRO 5. - OSTEOARTRITIS MENINGOCOCKCAS

\begin{tabular}{|c|c|c|c|c|}
\hline Aatores & Edad & Articulación & Tratamiento & Resuitado \\
\hline Netter 1899 & I4 años & bombro & $\begin{array}{l}\text { artrotomía } \\
\text { (Kirmisson) }\end{array}$ & anquilosis \\
\hline $\begin{array}{l}\text { Sainton } 7 \\
\text { Bosquet } 1916\end{array}$ & 35 anjos & $\begin{array}{l}\text { hombro } \\
\text { rodilla }\end{array}$ & stivero intraarticelar & anquilosis \\
\hline Roger 1918 & soldado & $\begin{array}{l}\text { cadeza } \\
\text { rodilla }\end{array}$ & $\begin{array}{l}\text { seroterapis, punción } \\
\text { articular, extensión } \\
\text { continua, yeso, } \\
\text { movilización bajo } \\
\text { anestesia }\end{array}$ & anquilosis \\
\hline $\begin{array}{l}\text { Herrick } y \\
\text { Parkhurst } I 919\end{array}$ & & muñcea & & $\begin{array}{l}\text { necrosis extremi- } \\
\text { daud distal del } \\
n \in \mathbb{1} \text { dio }\end{array}$ \\
\hline \multirow{3}{*}{ Sthein 1938} & 17 años & $\begin{array}{l}\text { codo } \\
\text { cadera }\end{array}$ & & arkquilosis \\
\hline & 19 años & $\begin{array}{l}\text { muneca } \\
\text { codo }\end{array}$ & seroterapia, geso & anquilosis \\
\hline & 48 años & $\begin{array}{l}\text { cadesa } \\
\text { rodilipa }\end{array}$ & $\begin{array}{l}\text { seroterapia. Feso, } \\
\text { extensión continua } \\
\text { diatermía }\end{array}$ & anquilosis \\
\hline
\end{tabular}

CUADRO 6. - ARTRITIS MENINGOCOCICAS PRLIMIRIAS O ORIPTOGENETICAS

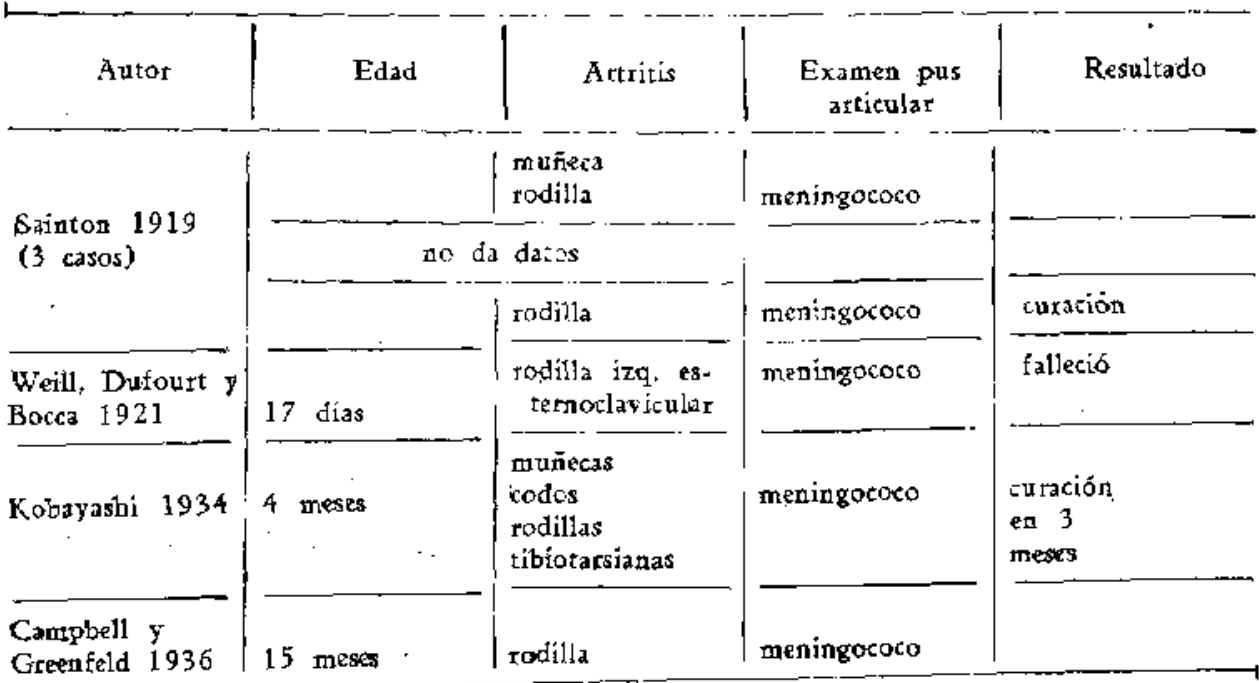


La artritis meningocócica se observa en todas las edades y con frecuencia más o menos igual en ambos sexos. El caso más precoz to constituye un lactante de $1 \mathrm{r} / 2$ mes, en el cual en una autopsia se encontraton artritis purulentas múltiples; sin embargo, en el primer año de vida la artritis meningocócica es algo menos frecuente, presentando en los años suce sivos variaciones escasas (Cuadro 7).

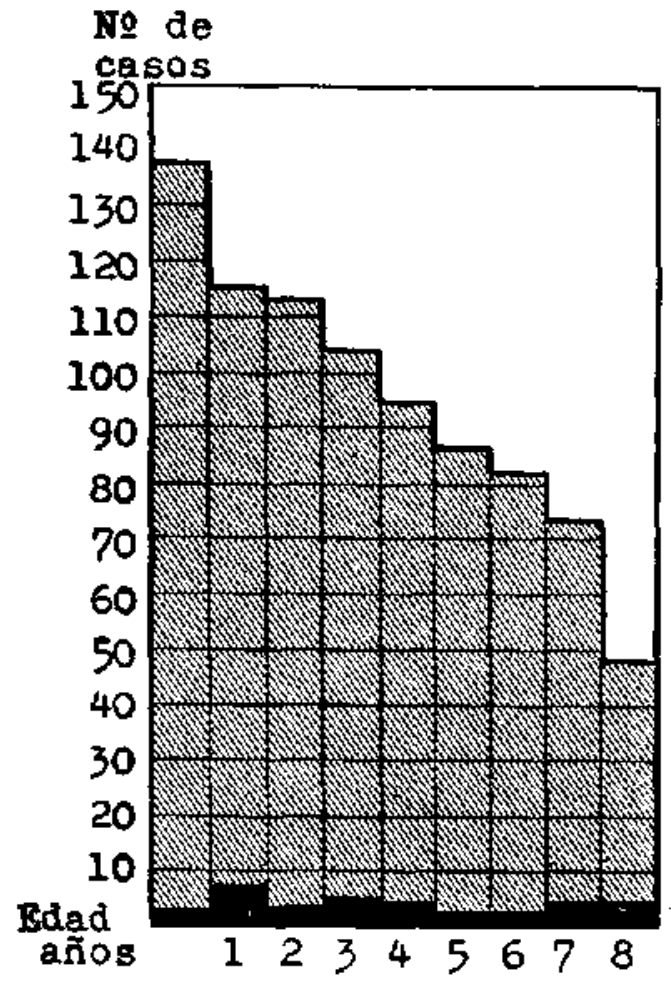

Cuadro 7. - Frecuencia co la artritis mentngocó izas per edadzs, en 1 , C00 casos le infecrion meningeóci: 1 .

Las pequiñas columuas nę:as rẹresentan los casos son artritis. Los niños mayores de 8 años han sido hospitalizados en parte en Serviclos de adultos, por lo cual se han eliminado en :s:e diagrama.

La articulación que se ha comprometido con más frtcuencia en nuestros casos, ha sido la muñeca. En cambio, en las series de Sainton y de Schein ha sido la rodilla (Cuadro 8). Según Rolleston, en los niños pequeños el compromiso de 
CUMDRO 8. - COMPROMISO ARTICULAIR POR ORDEN DE PRECUENCIA DIAGNOSTICO OLINTCO

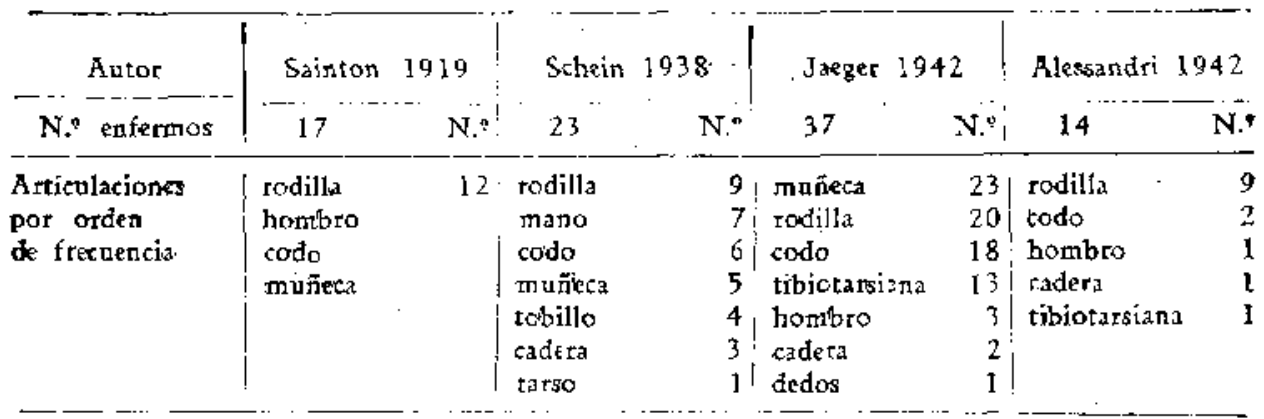

las pequeñas articulaciones de las manos y pies sería relativamente frecuente: sin embargo, nosotros lo hemos observado una sola vez, en un niño de 4 meses.

El compromiso mono y poliarticular lo hemos visto casi con igual frecuencia:

Monoarticular

Poliarticular

20 casos

2 articulaciones

17 "

3 articulaciones

5 "

4 articulaciones

6 ,

5 articulaciones

6 articulaciones

7 articulaciones

En las poliartritis generalmante una articulación está más comprometida que las demás.

Se dice que las artritis duran término medio 8 a 14 días. pero hemos visto casos que han desaparecido completamente a los 4 dias, $y$ alrededor de la tercera parte de nuestros 37 cascs han persistido más de 14 días. llegando algunos hasta los 2 meses. Como criterio de curación se ha considerado la desaparición absoluta de todo signo de compromiso articular.

Se inician generalmente con dolor más o menos intenso, el niño llora al tocarle o movilizarle la articulación comprometida. Al mismo tiempo, o uno o dos días después, se evidencia un aumento de volumen àrticular, que se acentúa en los 2 ó 3 días siguientes (Fig. 1). con aumento discreto de la temperatura local y cambio de coloración de la piel ligero 
T

o ausente. La escasa reacción de las partes blandas hacen pensar más bien en una hidartrosis y no en una piartrosis. Sólo en algunos casos (21\%) la aparición de la artritis va aconipañada o precedida de una recrudescencia de la curva febril. que se normaliza de ordinario en el curso de una semana; en cambio, no hemos podido establecer ninguna relación entre

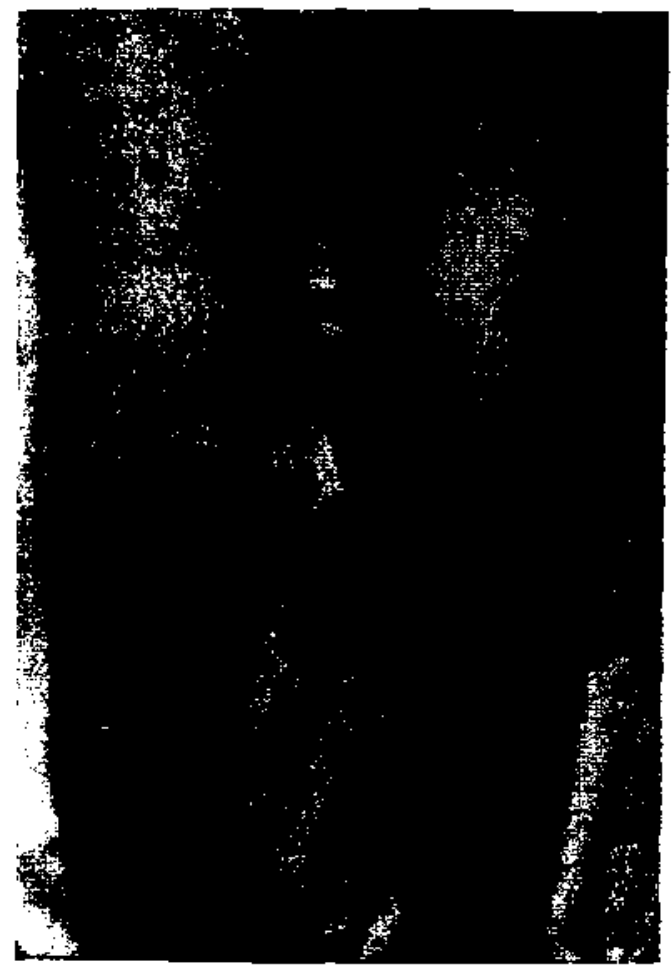

Figlira N. 1.

artritis y gravedad de la infección meningocócica. La intensidad del dolor es muy variable y muchas veces no existe relacion entre derrame y dolor: derrame abundante con dolor escaso $y$ viceversa, es decir, derrame escaso con dolor intenso; aunque no siempre es así y se observa a veces cierta telación entre la magnitud del derrame y la intensidad del dolor. El derrame suele ser intenso, comprobándose por ejemplo, en la rodilla choque rotuliano franco y repleción de los fondos de saco, derrame articular que, sin embargo, en el niño nenueño eutrófico es fácilmente enmascarado por el exuberante panícu- 
lo adiposo. La impotencia funcional está en relación con ta intensidad del dolor y del derrame, tomando el miembro, en los casos de derrame intenso, la típica actitud de Bonnet y observándcse otras veces verdaderas contracturas en flexión.

Muchas veces cuando existen signos subjetivos especialmente dolores fugaces, pero no objetivos de artritis meningocócica. se hace el diagnóstico de "artralgia". Sin embargo, el hallazgo anátomopatológico de sinovitis purulentas que no habian sido diagnosticadas intra vitam nos hace pensar que aun en estos casos existe un substrátum anatómico que justifique el término "artritis".

Junto con disminuir y desaparecer el aumento de volumen y el dolor. mejora la movilidad articular. Cuando ya prácticamente no existe dolor, en algunos casos se ve persistir durante largos días y aun semanas, infiltración periarticular comprobable a la palpación, manifiesta sobre todo en los fondos de saco de la rodilla y que sólo lentamente desaparece.

Punción articular.-En 4 casos hemos puncionado la rodilla y en uno la radiocarpiana, extrayendo en todos pus amarillo citrino, espeso y grumoso, con grumos de fibrina que a veces dificultaban la aspiración. Este es el aspecto que presenta el pus después de varios días de evolución. pues al comienzo es de un color de pasto verde pálido, viscoso y uniforme. y es así cómo lo hemos encontrado en las articulaciones en los niños fallecidos precozmente. En todos los casos el derrame se reproducía rápidamente mostrando la articulación en general al dia siguiente el mismo aspecto. En ningún caso se ha producido fistulización subsiguiente a la punción. En general, hemos sido parcos con las punciones, por una parte por tratarse de niños. en los cuales el espacio articular es pequeño y las epífisis aun cartilaginosas, $y$ por otra, por ser el pus espeso y grumoso, lo cual requería trócares de cierto calibre.

En el pus se encuentran abundantes polinucleares degenerados, algunas células endoteliales y aumento de proteína; el examen directo y el cultivo del pus ha sido negativo en nuestros 5 casos: esto se explica quizás por la acción de las sulfamidas, pues en la época presulfamidica los resultados eran positivos en la tercera parte o la mitad de los casos.

Radiología.-. En 8 casos se han radiografiado diversas articulaciones. tomando las articulaciones simétricas en und placa en la proyección anteroposterior para tener un medio de comparación. Pero siempre han sido negativas. Ya dijmos que se han publicado algunos pocos casos de osteoartri- 
tis meningocócica en los cuales la radiografía revelaba destrucción osteocondral y a veces subluxaciones patológicas.

Ciagnóstico.-El diagnóstico de las artritis que aparecen durante o después de la evolución de una meningitis meningocócica dificilmente se prestará a dudas. Sin embargo, en ciertas regiones deberá hacerse el diagnóstico diferencial con las tendosinovitis o bursitis meningocócicas supuradas. En canbio. en las artritis meningocócicas criptogenéticas la especificidad podrá sospecharse si simultáneamente se presentan otros casos de infección meningocócica, pero sólo podrá afirmarse con la demostración del meningococo en el pus articular. cascs excepcionales por lo demás.

Frcnóstica.--En los enfermos que presentan una artritis agregada a su cuadro meníngeo, tanto el pronóstico quoał vitam cuanto al pronóstico quoad functionem, después de los primeros días. en general es bueno y la resolución es la regla.

Todos nuestros casos han termínado con la, resolución completa del proceso articulat y sin dejar secuelas. 11 casos han sido controlados 2 a 8 meses después de ser dados de alta, gracias al Servicio Social y en todos ellos la recuperación aná. tomotuncional era completa. Por cierto que se trataba de niños. pues los autores concuerdan en que en éstos el pronóstico c en general mejor que en los adultos, en los cuales se han oterrvado casos. aunque raros, que después de un cuadro agudo, sin derrame apreciable, han llegado rápidamente a la anquilosis. pese a múltiples tratamientos ortopédico-quirúrgicos. Pere aun en los niños las artritis tienen importancia médicoeconómica. pues prolongan la hospitalización y exigen control médico continuado. por el hecho de persistir más allá del Ferícdo de estado de la meningitis.

Tratamiento.-El tratamiento de la infección meningocócica misma es è del dominio del internista o pediatra. Todos nuestros casos han sido tratados con los derivados sulfamidados actualmente en uso (sulfapiridina y sulfadiazina) administrados por las vías y a las dosis usuales. Pero las artritis no son influídas en absoluto por las sulfamidas; al contrario. en la mayoría de los casos se han iniciado. como se desfrende del cuadro 1, durante el tratamiento sulfamidado. en enfermos a los cuales se les había administrado ya grandes dosis de sulfamida, a veces durante 10 y más días antes de iniciarse la artritis. Rundiett et al. ya habian becho esta misma cbservación. En 1 caso en el cual después de vaciar el empiema articular inyectamos sulfapiridina soluble en la cavidad irticular, el derrame se reprodujo con igual rapidez y no se 
observó ninguna modificación favorable. Esto está de acuerdo con hechos comprobados en otros servicios (Alessandri y colaboradcres): que la artritis no cede, pese a que la concentración de sulfamida en el líquido articular es superior a la sanguinea, determinaciones que nosotros no hemos podido realizar por carecer de los elementos pertinenres.

La sueroterapia intraarticular $(10 \mathrm{cc}$.) y la medicación salicilada tampoco han dado resultados convincentes.

Los tratamientos quirúrgico-ortopédicos no han sid:s más afortunados. En todos los casos en los cuales hemos vaciado el derrame por punción y aspiración articular, aquél se reprodujo rápidamente, aun después de una segunda punción.

La inmovilización con aparatos de yeso o por extensión continua tampoco acelera la resolución de la artritis, al contrario, una vez retirado el aparato, persistía generalmente cierta rigidez articular que en 2 casos tuvo que ser sometida a quinesiterapia subsiguiente. Como los enfermos por el dolor esfontáneamente mantienen inmóvil su articulación. creemos que en general la inmovilización es superflua a no ser que por la intensidad del proceso sean de temer lesiones graves (osteoartritis) que pcr. la inmovilización trate darse alivio en casos muy dolorosos o que quiera vencerse por extensión continua una contractura en flexión. Alesandri et al. dicen haber obtenido beneficio con la aplicación de ultratermia $y$ sollux.

En las artritis meningocócicas puras, sin infección secundaria, creemos que la artrotomía que algunos han practicad: no encuentra indicación.

En las osteoartritis meningocócicas tal como en las lesiones crteoarticulares destructivas similares, el tratamiento ortopédicc deberá individualizarse en cada caso.

Vemcs, pues, que desgraciadamente las artritis meningocócicas evolucionan sin preocuparse por los medios terapéuti-. cos ensayados hasta abora y queda aquí labor fructífera por realizar.

\section{Resumen}

Se describen 37 casos de artritis meningocócica diagnosticadas clínicamente, de un total de 1,000 casos de infeccićn meningccócica $(3.7 \%)$. La frecuencia real de las artritis es mucho mayor pues en la mesa de autopsia en 52 casos se encontraron 13 con sinovitis purulentas, que no habian sido diagnosticadas clínicamente. Esto tambien habla en favor de 
ana meningococemia precoz, es decir, de la invasión hemalógena simultánea de los tejidos meningeos y extrameníngeos.

Se describen dos nuevas localizaciones de la infeccion meningocócica a nivel del aparato iocomotor: las butsitis y las tendosinovitis supuradas.

Se propone la denominación meningocosis para designar la infección meningocócica en su totalidad, englobando bajo la dencminación meningocosis del aparato locomotor. las artritis, bursis y tendosinovitis meningocócicas.

Se discute la clasificación anátomoclínica de las artritis de Herrick y Parkhurst se afirma que las artritis meningocócicas en la inmensa mayoría de los casos sen sinovitis su'puradas y que las llamadas "artralgias" meningocócicas también son artritis.

La articulación que clínicamente se ha comprometido con más frecuencia ha sido la muñeca siguiéndole con escasa diferencia la rodilla. El compromiso mono y poliarticular se ba observado más o menos con igual frecuencia.

El pronóstico ha sido siempre bueno, pero se recuerda aue en la literatura se encuentran algunos casos de osteoartritis meningocócica que han llegado a la anquilosis.

Aun no existe un tratamiento que permita controlar la crolución de las artritis, no mostrando las sulfamidas ninguna acción. Si bien el pronóstico generalmente es benigno, un tratamiento eficaz sería de proyecciones médico-económicas. pues abreviaria la hospitalización y la atención médica.

\section{Summary}

37 cases of meningococcic artbritis were found among a 1000 cases of meningococcic infection $(3,7 \%)$. The real trecuency of arthritis being higher, for in a post-mortem examination made of 52 cases were found 13 cases of purulent synovitis not clinicaly diagnosed. This also shows early meningococcemia, that is the simultaneous hematogenous invasion of the meningeal and extrameningeal tissues.

Two new localisations of the meningococcic infection at the locomotion apparatus are described: the purulent bursitis and the purulent tendosynovitis.

The term meningocosis is proposed in order to designate the meningccoccic infection on the whole and the inflammation cf the joints. bursae and tendon sheaths are treated under the denomination meningocosis of the locomotion apparatus. The anatomoclinical classification of Herrick and Park- 
hurst is put in doubt and $j \mathrm{t}$ is assured that in most cases the meningococcic arthritis are purulent synovitis and that the so called "meningococcic arthralgias" are also in reality arthritis.

The wrists are the joints that clinicly are more frequently involved. The knee joint follows with small difference. The mono and polyarthicular involvement are observed nearly with the same frequency.

The prognosis are always good, but it is to be reminded that in the literature are communicated some cases of meningocciccic osteoarthritis that have ended in ankylosis.

Unfortunately we have no treatment that is able to master the course of the arthritis, for the sulfamide has not any action in this regard. Although the prognosis is often a goud one, a succesful reatment is desirable, for it would be of economical importance. The hospitalisation and the medical atenticis could be then shortened

BHBLIOGRAF1A

ALESSANDRI, H, FRIIJS, E.; KAFFMAVN, M.; ROESCHMANN, W'; ECHENFUE. F. y DUCC1. H. -- I.a meningoroccia. Rev. Med, Chile 71: $106-124$ (Fór.j 1943

ALESSANDRI. 1I, IRIT1S. E.: DUCC. H.: ROESCHMANN, W, $y$ QUESNEY. P. - Estudio sobre las coneintracicres de sulfamididos en la sangre $y$ lrquido célalo-raquideo en enfermos de meningococia, Rev. Med. Chile 71: 242-246 (Mar.) 194.3.

BLll.NG] ON, R. W. - Spcadylitis foljowing cerbro-spinal meningitis. Jour. A. M. A. 83: 683-686 (Aug). 1924.

BRINTON, D. - Carebrospinal fever. Williłns y Wilkin, Baltimore, 1941. COMROE, B. I. -- Arthritis and allied conditions, Lea y Febiger. Philade:phia, 1941.

HERRICK, $W, W$, and PARKHURST, G. M. - Meningococtus Arthritis. Am. Jour. of Med. Scien. 158: 47?-481 (Oct.) 1919: re1. en Jour. A. M. A. 73: 1392 (Nov,), 1919.

HODES, H, L. and STRONG, P. S. -- Treatment of meningococcic meningitis with sulfonanides. Jout. A. M. A. 119:691-694 (Jnne), 1942. HORWITZ, A. - Infección meningocócica en Chile. E1 Autor. Santiago. 1942 .

HORWITZ. A.; KRALJEVIC. R.: PERONI. J.; URZUA. H.; RISTOR! C.; SESNIC, R.; BOCCARDO, H.: DELGADO, J. y AYUB, V. La infección meningocócica dẹ adolescente $\mathrm{y}$ del adulto. Rev. Méd. Chile $71:$ 125-136 (Feb.) 1943. 
L.AFOSSE, P. - Manifestations articulaires dans la méningite cézébro-spinale. Bu.l. et Mém, de la Soc. Méd, des Hop. de París 39: 299-305 (avril), 1915

NEAL, J. B. - Meningotectic meningitis in children. Jour. A. M. A. 105: 568.570 (Aug.), 1935.

RCLLESTCN, H, - Lumleian Lectures of Cerebro-spinal fever. The Lancet 196: 541-549, 593-601 and 645-65.3 (Apr.), 1919.

RUNDLETT, E.; GNIASSI, A. M. and PRICE, P. - Meningococcic meningitis. Pronosric significance of the spinal fluid sugar. Joor, A. M. A. 119: 695-96 (Jine), 1942.

SAINTON. P. - La forme swbaigue de la pyarthrose méningococcique. Bull. et Mém. de la Soc. Méd. des Hop. de Paris 40: 35l-354 (mat.) 1916.

SAINTON, P. $\rightarrow$ Meningocoscal rheumatisan and arcbritis. The Lancet 196; $1080-82$ (jun.), 1919.

SAINTON, P. et BOSQUET, J. - Arthrite, méningococcique de l'éfaule a forme plastique anquilosante. Manifestations pulmonaires et méningite coniécutives. Atthrite dn genou post-méningitique. Conjontivite et iridochoroidic. Incidents sérotherapiques. Bull. et Mém, de la Sos. Méd, des Hop. de Pariș 40: 344-351 (mar.). 1916.

SCHEIN. A. J. - Articular manifestations of meningocoecic infections, Arch. cf Int. Med. 62: 963-978 (Dec.), 1938.

SMITHBLRN, K. C.; KEMPF, G. F.; ZERFAS. L. G. and GLLMAN. L. H. - Meningococcic meningitis. A clinics! study of one bundred and forty-fout epidemic tases. Jour. A. M. A. 95:776.80 (Sept.), 1930.

WAGHEISTEIN", J. M. - Sulfmilamide in the treatment of $106^{\circ}$ patients with meningococcic infections, Jour. A. M. A. 111:2172-74, (Der). 1938. 